\title{
ESTUDIO GLOBAL DE LOS RANKINGS UNIVERSITARIOS
}

\section{Introducción}

Los rankings universitarios o "tablas de posiciones" (league tables), que hace sólo 15 años eran una novedad, hoy son una característica normal en la mayoría de los países con grandes sistemas de educación superior. Fueron creados hace 20 años por US News and World Report con el objeto de satisfacer la necesidad de mayor transparencia y para contar con datos comparativos acerca de las instituciones de educación. Vilipendiados por los críticos, pero aceptados con gusto por los padres de los estudiantes, comenzaron a aparecer sistemas de rankings copiados unos de otros en todo el mundo, poco después de que se introdujeran las matrículas pagadas o de que éstas se incrementaran. En todas partes donde ha surgido una clasificación, ésta ha sido recibida con una mezcla de entusiasmo por parte del público y con inquietud en las instituciones.

Una de las principales causas de esta preocupación es la tendencia de estos esquemas de ranking institucional a usar agregados ponderados de los indicadores para alcanzar un "puntaje" de calidad que lo abarque todo, lo cual, a su vez, permite a las instituciones compararse unas con otras. Al seleccionar un conjunto de indicadores en particular y asignar a cada uno una determinada ponderación, los autores de estos rankings imponen una definición específica de calidad a las instituciones que están clasificando. El hecho de que pueda haber otros indicadores legítimos o combinaciones de indicadores, por lo general se omite silenciosamente.

Curiosamente, sin embargo, hay poco acuerdo entre los autores de estos indicadores en cuanto la definición de "calidad". Los principales sistemas de ranking del mundo tienen muy poca relación 
entre ellos, si es que la tienen, y usan indicadores y coeficientes de ponderación muy distintos para llegar a una medida de calidad.

En este documento analizamos 17 sistemas de clasificación universitaria y sistemas de ranking en el mundo. Catorce son clasificaciones "nacionales" (Australia, Canadá, China, Hong Kong, Italia, Polonia, España, el Reino Unido y Estados Unidos) y tres son "internacionales" o "transnacionales". Específicamente, comparamos estas clasificaciones en términos de sus métodos de recolección de datos y sus indicadores de selección y ponderación. Observamos, asimismo, dieciocho sistemas de clasificación (el sistema alemán CHE) que no se ajustan a las "reglas" de la clasificación universitaria normal.

\section{II. ¿Qué son los rankings universitarios y las tablas de posiciones universitarias?}

Los rankings universitarios son listas de ciertas agrupaciones de instituciones (por lo general dentro de una sola jurisdicción nacional), clasificados en forma comparativa de acuerdo con un conjunto común de indicadores en orden descendente. Se presentan habitualmente en forma de "tabla de posiciones", muy parecida a la clasificación de los equipos deportivos en una sola división, de mejor a peor, según el número de triunfos y derrotas que han experimentado ${ }^{1}$.

Otro aspecto que cabe destacar es que, en su mayoría, son creadas por empresas de publicidad comercial. En parte, esto refleja el hecho de que los rankings comparten algunas de las características con las "guías de consumo" de diversos productos. Aun cuando no son guías para instituciones específicas, los editores de folletos institucionales pueden incorporar los datos del ranking como material complementario, con el objeto de proporcionar más información a sus

1 El término deriva de la tabla de posiciones usada en el Reino Unido que frecuentemente se comparaba con la Primera Liga (División) de la clasificación de los equipos de soccer profesional o fútbol de Inglaterra durante los años 1990, y que hoy en día se da en una amplia variedad de contextos en Gran Bretaña. 
lectores. El objeto de los rankings -al menos en teoría- es "ver lo que hay debajo" de un producto complejo. En muchos casos, el esfuerzo que se necesita para recopilar, juntar y analizar los datos necesarios para confeccionar los rankings es tan grande que su producción para cualquier otro fin no comercial es probablemente imposible.

Los sistemas de ranking universitarios pueden ser institucionales o subinstitucionales, y realizados a escala nacional o internacional. Los sistemas de ranking nacionales son aquellos con los que se miden casi todas las universidades de un país para comparar unas con otras. Así era el formato de ranking universitario original (por ejemplo, el creado por el US News and World Report, en 1981, ampliamente copiado en otros países). En la mayoría de los casos se comparan todas las universidades dentro de un país -sobre todo en Canadá (Maclean's Magazine) y Estados Unidos (US News and World Report) - aun cuando, en algunos casos, están divididas de acuerdo con ciertas características institucionales y sólo se confrontan con otras instituciones de características similares. Efectivamente, se ha creado un grupo perteneciente a una "mini" tabla de posiciones. Son los rankings de este tipo los que se incluyen en este informe.

Los sistemas globales de clasificación institucional son una nueva variación de la idea más antigua de los rankings nacionales. Actualmente hay sólo dos: el Academic Ranking of World Universities, de la Universidad Jiao Tong de Shanghai, que se publicó por primera vez en 2003, y el World University Rankings, del Times Higher Education Supplement (THES) de Gran Bretaña, publicado por primera vez en noviembre de 2004. El primer ranking internacional -pero no global- fue realizado en 1997 por la revista Asiaweek, que clasificó las universidades más importantes. En este informe también se cubren estos tres rankings.

Los rankings subinstitucionales comparan determinadas unidades universitarias con otras similares de otras instituciones. Por lo general, sólo abarcan instituciones nacionales y se refieren a ciertas escuelas profesionales, tales como Economía, Derecho y Medicina. Las 
escuelas de posgrado en Economía también son objeto de numerosas clasificaciones internacionales realizadas por The Economist, Financial Times, Wall Street Journal y Business Week. Estos tipos de tablas no están incluidas en este informe, porque, sencillamente, hay demasiadas para analizarlas en detalle. Sin embargo, al final de este documento estudiaremos una variación especializada en un tema específico (los rankings $\mathrm{CHE}$ ).

Hay también algunos que enfocan determinados aspectos de las actividades universitarias. Por ejemplo, Best American Research Universities clasifica las instituciones norteamericanas en cuanto a su rendimiento, al igual que, de una manera algo más burda, lo hace el Centre for Science and Technology Studies de Berna, Suiza, con sus cuadros de "Champions League" (Liga de Campeones). Asimismo, la revista Yahoo Magazine ha clasificado algunas universidades por su "conectividad", y el Journal of Black Higher Education lo ha hecho según su capacidad para integrar estudiantes de diferentes orígenes en sus tablas de diversidad étnica. Estos tipos no se han incluido en este estudio porque sus objetivos son mucho más específicos y limitados que los sistemas de clasificación general en los que deseamos concentrarnos.

\section{III. ¿Cómo funcionan los rankings y las tablas de posiciones?}

Las tablas de posiciones, por su naturaleza, están destinadas a reducir el trabajo de instituciones enteras a indicadores numéricos únicos y susceptibles de ser comparados. En la mayoría de los sistemas, es un proceso que consiste en tres etapas: primero, se recopilan los datos en indicadores; luego, se otorga un puntaje a los datos de cada indicador, y, en tercer lugar, los puntajes de cada indicador se ponderan y se suman.

Todos los sistemas de ranking operan mediante la comparación de instituciones respecto de una gama de indicadores. El número de éstos en un sistema de clasificación puede variar de manera 
significativa: desde cinco, en el caso más simple (el THES World Rankings), hasta varias docenas, en los más complejos (La Repubblica o Wuhan). Por lo tanto, algunas áreas específicas de actividad o tipos de rendimiento institucional se pueden comparar, entre las instituciones, de manera muy parecida a como se hace con los indicadores de desempeño.

Salvo unas pocas excepciones (sobre todo los rankings Excelencia de España), toman luego los datos en cada indicador y los convierten en una "puntaje". Habitualmente, esto se hace dándole a la institución con el puntaje más alto en un determinado indicador un puntaje perfecto de 100 y luego se otorgan puntajes más bajos a otras instituciones, basándose en lo cerca que estuvieron de la primera. Una vez que se han otorgado notas para cada indicador, éstas se ponderan, y la ponderación más alta se entrega a los indicadores que se estima de mayor importancia. Luego, las notas ponderadas de todos los indicadores se cuadran para obtener un puntaje final unificado para cada institución.

Evidentemente, la elección de los indicadores y la ponderación para cada uno produce una enorme diferencia en el resultado final. No es una exageración decir que cuando los editores publicitan su producto como la guía "de las mejores" instituciones, son ellos mismos los que, en general, deciden cuál es la mejor, simplemente a través de su elección de los indicadores y coeficientes de ponderación. En efecto, el acto de elegir un conjunto de indicadores y coeficientes de ponderación impone una misma definición de "calidad".

\section{La base probatoria de las tablas de posiciones. ¿Cómo se recopila la información?}

Una cuestión esencial en la preparación de las tablas de posiciones y rankings es el método para recopilar la información. Básicamente, hay tres fuentes de datos sobre instituciones:

- Datos de encuesta. Es posible usar las encuestas de opinión o experiencias de diversos interesados para obtener datos 
comparables sobre distintas instituciones en cuanto a la calidad de la educación.

- Terceros independientes. Frecuentemente, los organismos gubernamentales recopilan y publican información sobre instituciones en su jurisdicción, y esto se puede usar como un criterio objetivo para compararlas. Estos datos frecuentemente son de naturaleza financiera y están basados en antecedentes administrativos de los organismos que otorgan subvenciones.

- Fuentes universitarias. Son las más completas y detalladas.

El uso de cada fuente tiene sus pros y sus contras. Los datos de las encuestas son científicos, en el sentido de que registran en forma muy exacta las observaciones, pero, en la medida en que son usadas para encuestar a empleados o formadores de opinión en el valor de los títulos de varias instituciones, los críticos pueden, con justa razón, poner en duda el valor de dichas observaciones, ya que muy pocos empleadores o formadores de opinión tienen, supuestamente, una visión detallada o un conocimiento de cada una de las estudiadas. Las encuestas de estudiantes y personas recién graduadas también son criticadas porque, a pesar de que ellos pueden expresar sus sentimientos acerca de su propia institución, no tienen una base para compararla con otras.

Los datos administrativos proporcionados por terceros independientes (casi siempre gobiernos u organismos que otorgan subsidios) se consideran, habitualmente, "la regla de oro" de los datos comparativos, puesto que, al menos en teoría, son tanto exactos como imparciales. El problema es que -casi siempre- estos datos no son recopilados con el objeto de preparar "tablas de posiciones" sino, más bien, como un subproducto administrativo del trabajo rutinario. En consecuencia, confiar demasiado en esta fuente puede llevar a una situación en la cual los indicadores se eligen simplemente porque los datos están disponibles y no porque contribuyen a una definición acertada de calidad. 
En último término, hay datos proporcionados por las propias universidades. En algunos casos, cuando no se pueden obtener indicadores importantes de calidad a través de encuestas o terceras personas, los autores de los esquemas de ranking les envían cuestionarios a las mismas universidades. El beneficio de este enfoque es que se puede, teóricamente, responder un número de preguntas acerca de la calidad que de otra manera sería imposible. El principal inconveniente es que no existe garantía alguna de que las instituciones vayan a proporcionar datos verídicos al evaluador, ya que todas tienen el claro incentivo de manipularlos de modo que las pueda beneficiar. Efectivamente, en algunas instituciones de Estados Unidos existe personal -dentro de las oficinas de investigación institucional- cuya obligación es proporcionar datos favorables en las encuestas de US News and World Report.

En la tabla 1 se muestra la medida en que cada sistema de ranking usa cada fuente de datos.

Tabla 1. Número de indicadores por tipo de fuente de información.

\begin{tabular}{lcccc}
\hline & $\begin{array}{l}\text { Raw indicator } \\
\text { count }\end{array}$ & $\begin{array}{l}\text { Survey } \\
\text { data }\end{array}$ & $\begin{array}{l}\text { Third } \\
\text { parties }\end{array}$ & Universities \\
\hline $\begin{array}{l}\text { Asiaweek-Asia's Best Universities } \\
\text { (defunct, 2000) }\end{array}$ & 18 & - & - & 18 \\
\hline Daily Telegraph (2003) & 1 & - & 1 & - \\
\hline Education18.com & 9 & 3 & 4 & 2 \\
\hline Excelencia, 2001 & 71 & - & 71 & - \\
\hline Financial Times (2003) & 17 & - & 17 & - \\
\hline $\begin{array}{l}\text { Guangdong Institute of Management } \\
\text { Science }\end{array}$ & 17 & - & 14 & 3 \\
\hline Guardian-University Guide 2005 & 7 & - & 2 & 5 \\
\hline La Repubblica & 23 & 2 & 21 & - \\
\hline Maclean's University Rankings & 24 & 1 & 5 & 18 \\
\hline $\begin{array}{l}\text { Melbourne Institute- International } \\
\text { Standing of Australian Universities }\end{array}$ & 26 & 3 & 23 & - \\
\hline Netbig, 2004 & 18 & 1 & 10 & 7 \\
\hline Perspektywy / Rzeczpospolita Uniwersytet & 18 & 1 & 2 & 15 \\
\hline & & & & Continúa
\end{tabular}




\begin{tabular}{|c|c|c|c|c|}
\hline $\begin{array}{l}\text { Shanghai Jiao Tong University-Academic } \\
\text { Ranking of World Universities }\end{array}$ & 6 & - & 6 & \\
\hline The Times-Good University Guide 2005 & 9 & - & 9 & - \\
\hline $\begin{array}{l}\text { Times Higher Education Supplement } \\
\text { - World University Rankings }\end{array}$ & 5 & 1 & 1 & 3 \\
\hline $\begin{array}{l}\text { US News and World Report-America's } \\
\text { Best Colleges } 2006\end{array}$ & 15 & 1 & 3 & 11 \\
\hline $\begin{array}{l}\text { Wuhan University Centre for Science } \\
\text { Evaluation }\end{array}$ & 45 & 2 & 22 & 21 \\
\hline
\end{tabular}

Fuente: Los autores

La tabla anterior muestra que las encuestas son las fuentes de datos que se usan con menos frecuencia para los indicadores. Por cierto, sólo los rankings de Education 18, de Hong Kong, se acercan a tener una pluralidad de indicadores provenientes de esta fuente. Sin embargo, hasta cierto punto esta medida subestima la importancia de las encuestas, porque no explica la ponderación que se le otorga a cada indicador en cada estudio. En el THES World Rankings, por ejemplo, hay una sola encuesta (para los efectos de la "reputación"), pero sólo corresponde al 50\% del ranking total. Asimismo, los rankings Maclean's de Canadá tienen un solo indicador basado en encuesta de un total de 24, pero éste vale un 20\% del puntaje final.

Fuera de Norteamérica, las fuentes de terceros son lejos las más usadas: cuatro de los 18 esquemas de ranking nombrados aquí las usan en forma exclusiva. En los 14 restantes, las fuentes de terceros comprenden una pluralidad de indicadores en ocho y las fuentes universitarias forman una pluralidad en seis. El predominio de datos de universidades se comprende muy bien en los casos de los rankings de Asiaweek y THES, ya que su alcance internacional reduce en forma significativa la posibilidad de que fuentes de terceros proporcionen datos sobre una base transnacional coherente ${ }^{2}$. En los casos de US News and World Report, Maclean's y el Guardian y Rzeczpospolita la explicación parece ser que no se pueden operacionalizar las

2 Shanghai Jiao Tong, el tercer estudio internacional en esta comparación, solucionó este problema confiando casi exclusivamente en las medidas de los resultados de investigaciones, tales como publicaciones científicas y citas. 
definiciones del término "calidad" -dadas por los editores- usando datos administrativos del gobierno. Esto podría indicar un fracaso en la recopilación de datos del gobierno en estos países, en cuanto la información que se considera importante para medir la calidad no se reúne en forma coherente ni centralizada; o, alternativamente, que los puntos de vista de los que crean los rankings sobre qué es lo que constituye un indicador de calidad no son compartidos por los gobiernos o la comunidad de educación superior.

\section{Lo que miden las tablas de posiciones. Una mirada a los indicadores y coeficientes de ponderación}

No debería ser una sorpresa enterarse de que los distintos sistemas de ranking usan indicadores muy diferentes para obtener una impresión de la "calidad". El número de indicadores individuales usados en los sistemas de ranking en todo el mundo ascienden fácilmente a unos cientos, lo que hace que cualquiera cuadrícula de comparación sea demasiado grande para ser de utilidad.

Para observar los indicadores y los coeficientes de ponderación de una manera manejable los hemos catalogado en siete títulos más grandes, basados, en parte, en un modelo de calidad institucional existente. En su proposición de un sistema para medir la calidad en la educación postsecundaria, Finnie, Ross y Usher (2005) desarrollaron un marco conceptual basado en los elementos siguientes:

1. Características iniciales: representan los atributos y aptitudes de los estudiantes en el momento de comenzar sus programas.

2. Aportes al aprendizaje:

a) recursos, tanto financieros como materiales, de que disponen los estudiantes y la facultad para los fines educacionales, y

b) personal docente, tanto en términos de número como en la manera de enseñar y el ambiente de aprendizaje que crean, medidos por la cantidad de tiempo que tienen los alumnos para estar en contacto con sus profesores, los tipos de exámenes que deben enfrentar, entre otros factores. 
3. Rendimiento en el aprendizaje: representa el "conjunto de aptitudes" $u$ otros atributos de los que terminan su experiencia educacional, tales como pensamiento crítico, razonamiento analítico y conocimientos técnicos. También incluye los registros relacionados con la retención de alumnos y el término de los estudios.

4. Resultados finales: representan los fines fundamentales a los cuales puede contribuir el sistema educacional, no sólo aquellas medidas tradicionales como las tasas de empleo e ingresos, sino cualquier otra considerada importante para las personas y la sociedad, tales como satisfacción en el trabajo, ser "un buen ciudadano", entre otras.

Estos cuatro elementos o categorías abarcan, en realidad, la mayoría de los indicadores usados por los sistemas de ranking tratados en este estudio. Sin embargo, vamos a modificar la tipología de dos maneras: primero, vamos a hacer una clara distinción entre recursos financieros y personal docente, y, segundo, incluiremos otros dos conjuntos de indicadores, a saber, "investigación" y "reputación".

No obstante, los rankings son, más que una simple colección de indicadores, una suma ponderada de éstos. Por lo tanto, es importante ver cómo se reúnen y cómo cada sistema de ranking define, implícitamente, la calidad educacional a través de la distribución de su ponderación. Aun cuando las diferencias aparentes entre los sistemas de ranking son importantes, hay algunas similitudes reales y curiosas entre algunos conjuntos de tablas de posiciones.

La tabla 2 muestra las diferencias entre los indicadores y los coeficientes de ponderación usados por los distintos sistemas. Cada fila de la lista resume la distribución de los coeficientes de ponderación del indicador entre las siete categorías de indicadores descritas en la sección anterior y que suma $100 \%$. Es obvio, incluso desde la mirada más superficial de esta tabla, que no hay dos sistemas de ranking iguales y, en efecto, algunos prácticamente no tienen áreas que se superpongan unas con otras. 
Tabla 2. Ponderaciones de las tablas de posiciones

\begin{tabular}{|c|c|c|c|c|c|c|c|}
\hline Publication & \begin{tabular}{|l|} 
Beginning \\
characteristics
\end{tabular} & $\begin{array}{l}\text { Learning } \\
\text { inputs - staff }\end{array}$ & $\begin{array}{l}\text { Learning inputs } \\
\text { - resources }\end{array}$ & $\begin{array}{l}\text { Learning } \\
\text { outputs }\end{array}$ & $\begin{array}{l}\text { Final } \\
\text { outcomes }\end{array}$ & Research & Reputation \\
\hline $\begin{array}{l}\text { Asiaweek (Indial } \\
\text { Asia) }\end{array}$ & 25 & 28.3 & 10 & 0 & 0 & 16.7 & 20 \\
\hline $\begin{array}{l}\text { Daily Telegraph } \\
\text { (UK) }\end{array}$ & 0 & 100 & 0 & 0 & 0 & 0 & 0 \\
\hline $\begin{array}{l}\text { Education18.com } \\
\text { (Hong Kong) }\end{array}$ & 20 & 15 & 5 & 0 & 0 & 20 & 40 \\
\hline Excelencia (Spain) & 0 & 25 & 25 & 25 & 0 & 25 & 0 \\
\hline $\begin{array}{l}\text { Financial Times } \\
(U K)\end{array}$ & 9 & 19 & 15 & 10 & 27 & 20 & 0 \\
\hline $\begin{array}{l}\text { Guangdong Institute } \\
\text { (China) }\end{array}$ & 0 & 0 & 0 & 57.1 & 0 & 42.1 & 0 \\
\hline $\begin{array}{l}\text { Guardian University } \\
\text { Guide (UK) }\end{array}$ & 28 & 35 & 10 & 10 & 17 & 0 & 0 \\
\hline La Repubblica (Italy) & 10 & 44.4 & 15.6 & 10 & 0 & 20 & 0 \\
\hline Maclean's (Canada) & 15 & 20 & 44 & 5 & 0 & 0 & 16 \\
\hline $\begin{array}{l}\text { Melbourne Institute } \\
\text { (Australia) }\end{array}$ & 11 & 3.5 & 11 & 12.6 & 4.8 & 40 & 17.1 \\
\hline Netbig (China) & 12 & 21.8 & 6 & 0 & 0 & 45.2 & 15 \\
\hline Newsweek (US) & 10 & 20 & 10 & 0 & 0 & 60 & 0 \\
\hline $\begin{array}{l}\text { Perspektywy / } \\
\text { Rzeczpospolita } \\
\text { (Poland) }\end{array}$ & 8 & 20.5 & 11.5 & 0 & 0 & 0 & 50 \\
\hline $\begin{array}{l}\text { Shanghai Jiao } \\
\text { Tong University } \\
\text { (Intl/China) }\end{array}$ & 0 & 0 & 0 & 10 & 0 & 90 & 0 \\
\hline $\begin{array}{l}\text { The Times Good } \\
\text { University Guide } \\
\text { (UK) }\end{array}$ & 3.3 & 53.3 & 6.7 & 3.3 & 3.3 & 30 & 0 \\
\hline $\begin{array}{l}\text { Times World } \\
\text { University Rankings } \\
\text { (UK) }\end{array}$ & 5 & 25 & 0 & 0 & 0 & 20 & 50 \\
\hline $\begin{array}{l}\text { US News and World } \\
\text { Report (US) }\end{array}$ & 15 & 20 & 15 & 25 & 0 & 0 & 25 \\
\hline Wuhan (China) & 10.6 & 8.5 & 16.6 & 3.4 & 0.6 & 48.6 & 11.7 \\
\hline
\end{tabular}

Fuentes: Los autores

Pese a las grandes diferencias en la elección de indicadores y coeficientes de ponderación que se constatan en todo el mundo, aparecen algunos modelos cuando los estudios se agrupan 
geográficamente. Por ejemplo, los estudios de China -que tiene cuatro proyectos de ranking distintos- colocan mucho más peso en los indicadores de investigación que cualquier otro estudio en el mundo. En el caso más extremo -el de Academic Ranking of World Universities, de la Universidad de Jiao Tong de Shanghai- el desempeño de la investigación vale un $90 \%$ del ranking total. Ello seguido por Wuhan, donde las medidas de investigación valen $48,2 \%$ de la tabla definitiva, Netbig $(45,2 \%)$ y Guangdong (42,1\%). Como hemos visto, gran parte de la ponderación proviene de contar papeles y citas de estudios bibliométricos -con un fuerte sesgo hacia las ciencias exactas. Con excepción de Guangdong, que enfoca sobre todo los resultados del aprendizaje (principalmente tasas de graduación), los sistemas chinos ponen también un importante énfasis en la fama institucional. Por el contrario, comparativamente se pondera poco el aporte de recursos o los resultados finales, ya sea porque los datos sobre estos temas son escasos o porque los expertos chinos consideran que estos indicadores no tienen importancia. Esta es una cuestión abierta.

Otros modelos regionales también son evidentes. Las tablas de las universidades del Reino Unido, por ejemplo, se abstienen de usar las encuestas de reputación como un medio para determinar la calidad (aun cuando THES le otorga una ponderación de 50\% a los temas de reputación/fama). Las británicas también ponen mucho más énfasis a las medidas del personal docente y a la calidad de éstos -en promedio, le dan más de un $40 \%$ de ponderación a esta área, frente a un promedio de sólo $5 \%$ en el resto de las tablas del mundo en su conjunto.

Las dos grandes encuestas norteamericanas-Maclean's y de US News and World Report-son prácticamente idénticas en la distribución de la ponderación, salvo el hecho de que la versión canadiense pondera mucho más el aporte de recursos y la estadounidense los resultados del aprendizaje (curiosamente, los coeficientes de ponderación de categoría general de los rankings de La Repubblica de Italia son muy similares en su naturaleza a los de Maclean's y de US News, aunque los indicadores específicos son completamente diferentes). 
La tabla 2 demuestra la premisa central de este estudio: los diferentes sistemas de ranking tienen definiciones de calidad muy distintas. En la educación superior, este concepto es, claramente, muy maleable: algunos observadores quieren enfocar los rendimientos, mientras otros se concentran en los aportes; por otra parte, existe poco acuerdo en cuanto a qué tipos de aportes y resultados son importantes. No sólo se prescinde de un indicador único a lo largo de todos los esquemas de ranking, tampoco hay una sola categoría de indicadores en común. Increíblemente, ninguna de las siete categorías básicas de indicadores son comunes para todos los sistemas de ranking universitario.

Uno de los estudios previos de las tablas de posiciones (Dill y Soo, 2004) llegó a la conclusión, sobre la base de un examen de cuatro conjuntos de tablas en cuatro países, que las definiciones internacionales de calidad eran convergentes. Nuestros hallazgos, basados en una muestra más amplia, contradicen sus resultados. Parte de la razón de esta contradicción reside en el hecho de que dividimos los indicadores en siete categorías en vez de cuatro y, por ende, siempre existía la posibilidad de que encontráramos más variaciones. No obstante las variaciones metodológicas $-\mathrm{y}$ creemos que nuestra metodología es la más refinada de las dos-, los resultados aún discrepan. Estimamos que si Dill y Soo hubiesen observado los esquemas de ranking asiáticos o internacionales, ellos también habrían encontrado estas diferencias y habrían corregido sus conclusiones.

\section{Coherencia de los resultados a lo largo de las tablas de posiciones}

Del análisis anterior se podría concluir que la calidad institucional medida no es inmutable y que el ranking de la institución es, en gran parte, una función de lo que el organismo que lo realiza decide medir. Un ejemplo es la Queen's University de Kingston, Canadá. En sus rankings nacionales (Maclean's) le va muy bien porque atrae a buenos estudiantes y está razonablemente bien dotada y bien fundada. En 
cambio, en los internacionales le va bastante mal, incluso comparada con otras universidades canadienses, porque es una universidad pequeña, lo que la pone en desventaja en términos de medición de resultados de investigación no normalizados.

Dada la plétora de sistemas de ranking que han aparecido en los últimos años, ahora uno puede poner a prueba esta proposición directamente. En la mayoría de los países, hay por lo menos tres "observaciones" de rankings efectuadas por diferentes sistemas nacionales e internacionales (los de THES y Shanghai Jiao Tong, más uno o más nacionales). En aquellos casos en que uno puede usar múltiples esquemas para observar los puntajes relativos de las instituciones en un solo país, nos encontramos que ciertas organizaciones invariablemente están en los primeros lugares: Oxford y Cambridge en el Reino Unido, Harvard, Yale, Princeton, MIT y Stanford en los Estados Unidos de Norteamérica; Peking y Tsinghua en China, y la Universidad de Toronto en Canadá. Pese a las diferencias entre los esquemas de ponderación y agregación usados por las tablas de posiciones nacionales e internacionales, estas instituciones logran monopolizar sistemáticamente los primeros lugares. Más abajo en la escalera ordinal, los diferentes sistemas de ranking empiezan a mostrar una mayor variación (esto es, raramente hay acuerdo entre los sistemas en cuanto a qué universidad ocupa el décimo lugar). Mas, a pesar del esquema usado, al parecer "estas universidades top" casi siempre resultan ser las mejores universidades.

Esto plantea un serio problema de interpretación. Si los rankings fueran absolutamente inconsistentes a lo largo de todas las tablas de posiciones, sería muy fácil desechar del todo su presencia como un ejercicio intelectual sin valor alguno y diseñado solamente para vender diarios o revistas. Por otra parte, si los rankings fueran absolutamente coherentes a lo largo de todas las tablas, entonces podríamos concluir que probablemente hay uno o dos "súper" indicadores que están manejando todas las tablas y que el resto es esencialmente "ruido" con qué distraer a los lectores y crear falsas diferenciaciones. Sin embargo, ninguno de estos escenarios es real; lo 
que aparentemente sucede es que los diferentes esquemas de ranking proporcionan resultados consistentes para algunas instituciones e inconsistentes para otras.

La explicación más simple es que las tablas de posiciones institucionales no miden lo que sus autores creen que están midiendo. Ellos creen que cada indicador es un representante razonable de la calidad y que, debidamente agregados y ponderados, constituyen una "definición" plausible, totalizadora (holística) de calidad. Efectivamente, la mayoría de los indicadores probablemente son epifenómenos de alguna característica subyacente que no se está midiendo. Es decir, hay algún "asunto oscuro" que está ejerciendo un tirón gravitacional en todos los esquemas de ranking de manera que ciertas instituciones o tipos de instituciones (las Harvards, las Oxfords y las Tsinghuas del mundo) se alzan a la cabeza cualesquiera sean los indicadores específicos y las ponderaciones usados. Indudablemente este "asunto oscuro" merece ser objeto de una investigación en el futuro. Nuestra suposición, sin embargo, es que "la edad de la institución", "el tamaño de la facultad" y "el gasto por estudiante" son probablemente excelentes candidatos para individualizar este "asunto oscuro".

\section{Rankings sin tablas de posiciones: el enfoque CHE}

En la mayor parte de este documento hemos estado describiendo las tablas de posiciones, es decir, los sistemas de ranking que proporcionan un puntaje único integrado que permite una clasificación ordinal de instituciones enteras. Sin embargo, este no es el único enfoque posible de los rankings de universidades. No hay, por ejemplo, ninguna razón intrínseca de por qué los indicadores deben enfocarse solamente en las instituciones; también es posible usar enfoques que las estudien en ámbitos administrativos más bajos (tales como departamentos o facultades). The Guardian y, a partir del 2006, la Repubblica proporcionan rankings globales departamentales en universidades enteras (es decir, proporcionan tablas separadas para cada disciplina), aun cuando también sintetizan los datos hacia 
arriba en rankings institucionales, como lo hemos expuesto en las dos secciones anteriores.

El Centre for Higher Education Development (CHE) de Alemania ha adoptado un enfoque totalmente distinto y publica rankings anuales, asociado con un medio de comunicación (actualmente Die Zeit, antes Stern). Regularmente, realiza encuestas a unos 130.000 estudiantes y a 16.000 docentes que abarcan casi 250 institutos de educación superior. La encuesta a los estudiantes formula preguntas sobre las experiencias de los estudiantes y su grado de satisfacción; la de los docentes está diseñada de manera que genere datos para un determinado indicador conocido como "la elección de uno de adentro" (le pide a los profesores que nombren tres instituciones en su especialidad de estudio que ellos recomendarían a alguien como los mejores lugares para estudiar). Además, tiene una cantidad de indicadores que usan fuentes independientes de datos. Aproximadamente dos tercios de los indicadores se basan en la encuesta (superior a cualquier tabla de posiciones señalada en este estudio) y los puntos de datos restantes provienen de fuentes de terceros. Los rankings CHE no utilizan datos de fuentes universitarias.

El ranking CHE de los departamentos de las universidades alemanas es distinto a las tablas de posiciones tradicionales de dos maneras sorprendentes. Primero, como decíamos, no pondera ni suma puntajes de indicadores individuales: todos los datos de departamentos, en cada indicador, se pueden considerar en forma independiente y no se intenta clasificar los departamentos en una escala ordinal. Ello porque estima que, desde un punto de vista optimista, no tiene sentido alguno (y desde el punto de vista pesimista puede ser muy desorientador) combinar indicadores ampliamente dispares en una sola jerarquía global.

Esta postura presenta ciertas dificultades para entregar los datos en formato impreso. En vez de una clasificación ordinal simple, hay que mostrar todos los indicadores para todas las instituciones, lo que significa que son algo complicados de manejar y difíciles de leer. Por 
otra parte, esta postura tiene una enorme ventaja cuando se traduce a la World Wide Web3.

Como el CHE no pondera los puntajes, los mismos usuarios pueden crear sus propias ponderaciones y rankings seleccionando un número limitado de indicadores y pidiéndole a la base de datos del sitio Web que le proporcione información institucional comparativa sobre esa base. Al hacer esto, el enfoque cede el poder de definir "calidad" -que es uno de los roles claves que se arrogan los autores de esquemas de ranking- a los consumidores del sistema de ranking (los futuros estudiantes y sus padres o auspiciadores).

El segundo punto exclusivo de CHE es que, incluso dentro de cada indicador, no se hace ningún intento por asignar clasificaciones ordinales. Cada departamento de una institución en una determinada disciplina se clasifica simplemente como ubicado entre los "tres mejores", los "tres intermedios" y los "tres más bajos" de todas las instituciones con respecto a un determinado indicador. Las escuelas dentro de estas tres categorías se consideran cualitativamente igual, sobre la base de que, para muchos indicadores, los rankings ordinales son espurios dada la pequeña diferencia en la medición.

\section{Conclusiones}

Sobre la base de este estudio acerca de tablas de posiciones, podemos concluir que:

1) Hay grandes diferencias entre las tablas en términos de lo que se mide, cómo se mide, y cómo definen implícitamente "calidad".

2) Algunas de estas diferencias son, al parecer, de naturaleza geográfica o cultural. Hay una notable agrupación de ciertos tipos de indicadores y fuentes de datos. No está claro si esto refleja auténticas diferencias de opinión sobre la definición de "calidad" en las universidades o diferencias transnacionales en

3 Los datos se pueden encontrar en http/www.daad.de/deutschland/studium/ hochshulranking/04690.en.html 
la recopilación y disponibilidad de datos, aun cuando nosotros nos inclinamos por la primera explicación. La falta de indicadores comunes entre los países explica por qué las grandes tablas internacionales (Shanghai Jiao Tong y THES) dependen tanto de medidas de publicaciones y de encuestas de reputación (respectivamente), ya que son indicadores que no dependen de la recopilación y procesamiento de datos hechos por gobiernos o instituciones.

3) Pese a las grandes inconsistencias en las metodologías usadas para clasificar las universidades, hay un sorprendente nivel de acuerdo entre los sistemas de ranking para clasificar "las mejores" universidades en un determinado país. En la medida en que las diferentes metodologías entregan opiniones diferentes acerca de la calidad de una institución, la variación entre las observaciones aumenta según se desciende por los rankings ordinales.

4) Aun cuando la definición de "calidad" es objetada, las tablas de posiciones por definición imponen el enfoque de uniformidad para el tema, esta es la razón de que sean tan controvertidas. Sin embargo, como lo demuestra el enfoque CHE, las tablas de posiciones no son la única manera de enfocar los rankings. En efecto, la propagación de la World Wide Web proporciona a quienes recopilan datos institucionales una oportunidad de democratizarlos y ponerlos en manos del consumidor, siguiendo el enfoque de uniformidad.

Como lo observó Merisotis (2002), los rankings universitarios llegaron para quedarse. Por imperfectos que sean, satisfacen una demanda pública de transparencia e información que gobiernos e instituciones no han podido satisfacer por sí mismos. Además, a medida que la educación universitaria se vaya haciendo más onerosa para las personas y las familias, la demanda de información comparativa sobre las universidades aumentará. Sin embargo, las tablas de posiciones, como un medio de entregar dicha información, sólo están en su infancia y todos se beneficiarían con un análisis más detallado sobre los supuestos implícitos en sus propios esquemas. Esto es especialmente cierto respecto de las tablas de posiciones 
internacionales, que han limitado el rango de los posibles indicadores debido a la falta de datos comparativos entre los países. En la medida en que los esquemas de rankings internacionales están asumiendo un papel para asegurar la calidad en el creciente mercado estudiantil internacional, la comunidad de educación superior global necesita comenzar a analizar cómo puede recopilar y entregar información para permitir comparaciones interinstitucionales serias y responsables.

\section{Referencias bibliográficas}

The Atlantic Monthly (2003) Our First College-Admissions Survey'. The Atlantic Monthly, November, pp. 104-106.

Confessore, Nicholas (2003) What Makes a College Good? The Atlantic Monthly, November, pp. 104-105.

Fallows, James (2003) The New College Chaos. The Atlantic Monthly, November, p. 106.

Ganeshananthan, W. (2003) The Late-Decision Program, The Atlantic Monthly, November, p. 116.

Mathews, Jay (2003) The Bias Question. The Atlantic Monthly, November, p. 130.

Peck, Don (2003) The Selectivity Illusion. The Atlantic Monthly, November, p. 128.

Clarke, Marguerite (2002) Some Guidelines for Academic Quality Rankings. Higher Education in Europe, XXVII(4), pp. 443-459.

Dill, David D. \& Soo, Maarja (2004) Is There a Global Definition of Academic Quality? A Cross-National Analysis of University Ranking Systems. Chapel Hill, NC: Public Policy for Academic Quality background paper, University of North Carolina.

Eccles, Charles (2002) The Use of University Rankings in the United Kingdom. Higher Education in Europe, 27(4), pp. 423-432.

The Economist (2005) The year of listing differently. September 24, p 81.

Education18. Available at http://education18.com

The Financial Times (2003) Financial Times Universities 2003, May 03.

Finnie, Ross \& Usher, Alex (2005) Measuring the Quality of Post-secondary 
Education: Concepts, Current Practices and a Strategic Plan. Kingston, Ont.: Canadian Policy Research Networks.

Gladwell, Malcolm (2005) Getting in: the social logic of Ivy League admissions. The New Yorker, October 10, p. 80.

The Guardian (Leach, Jimmy, editor; 2004). The Guardian University Guide 2005: What to study, where to go and how to get there. London: Atlantic Books.

Hicks, Diana (2004) The Four Literatures of Social Science. Handbook of Quantitative Science and Technology Research. Henk Moed: Kluwer Academic.

Jobbins, David. The Times/The Times Higher Education Supplement - League Tables in Britain: An Insider's View. Higher Education in Europe, 27(4), pp. 383-388.

Krueger, Alan B. \& Hanushek, Eric A. (2000) The Class Size Policy Debate, Working Paper $N^{\circ} 121$. Washington DC: Economic Policy Institute.

Maclean's (Johnston, Ann Dowsett, editor; 2005). Maclean's Guide to Canadian Universities '05. Toronto: Rogers Publishing Limited.

De Miguel, Jesús M.; Sánchez, Jara D. \& Vaquera, Elizabeth (2004) University Ranking in Spain: Methods for Evaluating Public and Private Institution. Presentation at UNESCO-CEPES Conference, Washington DC.

Melbourne Institute of Applied Economic and Social Research (Williams, Ross \& Van Dyke, Nina; 2004). The International Standing of Australian Universities. Melbourne: University of Melbourne.

Merisotis, Jamie P. (2002A). On the Ranking of Higher Education Institutions. Higher Education in Europe, 27(4), pp. 361-363.

Merisotis, Jamie P. (2002B). Summary Report of the Invitational Roundtable on Statistical Indicators for the Quality Assessment of Higher/Tertiary Education Institutions: Ranking and League Table Methodologies. Higher Education in Europe, 27(4), pp. 475-480.

Netbig Chinese Rankings Available at http://www.netbig.com

Le Nouvel Observateur (2003) Universités le palmarès 2003. March 13.

Perspektywy (2004) University Rankings. October 2004.

Pike, Gary R. (2004) Measuring Quality: A Comparison of US News Rankings and NSSE Benchmarks. Research in Higher Education, 45(2), pp. 193208. 
The Princeton Review. America's Best Value Colleges. Available at http://www. princetonreview.com

Provan, David \& Abercromby, Karen (2000) University League Tables and Rankings: A Critical Analysis. CHEMS Paper $\mathrm{N}^{\circ}$ 30. London: Commonwealth Higher Education Management Service (CHEMS), Association of Commonwealth Universities.

Push UK. The Push Guide to Universities in the UK. London: Nelson Thornes, 2004.

La Repubblica, October 2004.

Sebkova, Helena (2002) Accreditation and Quality Assurance in Europe. Higher Education in Europe, 27(3) pp. 239-247.

Siwiski, Waldemar (2002) Perspektywy-Ten Years of Rankings. Higher Education in Europe, 27(4), pp. 399-406.

Der Spiegel (2004, № 48) Where Do the Best Study? Elite Students at German Universities? November 22, pp. 178-200.

Teixiera, IC; Teixiera, JP; Pile, M; Durão (1997) Classification and Ranking of Higher Engineering Education Programmes and Institutions: The ICT View.

Terenzini, Patrick T.; Pascarella, Ernest T. (1994) Living with Myths. Change, 26(1), pp. 28-33.

O'Leary, John; Hindmarsh, Andrew \& Kingston, Bernard (2004) The Times Good University Guide 2005. Hammersmith, London: Times Newspapers Limited.

Van Dyke, Nina (2005) Twenty Years of University Report Cards Higher Education in Europe, 30(2), pp. 103-125.

The Washington Monthly College Guide (2005) The Washington Monthly, 37(9), pp. 24-37.

Wonicki, Jerzy and Morawski, Roman Z. (2002) Public and Private Higher Education Institutions-Joint or Separate Evaluation and Rankings: The Polish Perspective. Higher Education in Europe, 27(4), pp. 461-466.

Yonezawa, Akiyoshi; Nakatsui, Izumi \& Kobayashi, Tetsuo (2002) University Rankings in Japan. Higher Education in Europe, 27(4), pp. 377-382.

Recibido: 21 de septiembre de 2006

Aceptado: 5 de octubre de 2006 